\title{
Tunnelling in cyclocarbenes: An application of Semiclassical Transition State Theory in reduced dimensions
}

\author{
Timothy A. H. Burd, Xiao Shan, David C. Clary \\ Physical and Theoretical Chemical Laboratory, South Parks Road, Oxford, OX1 3QZ, United Kingdom
}

\begin{abstract}
Methods to calculate rates of chemical reactions ab initio, in reduced dimensions, using Semiclassical Transition State Theory have recently been formulated. Here we further develop its application to unimolecular reactions where the choice of active degrees of freedom is not clear a priori. We describe in more detail a procedure to determine which degrees of freedom must be accounted for explicitly in the reduced dimensionality framework, and illustrate its success on a hydrogen transfer reaction of a cyclocarbene species.

Keywords: Semiclassical Transition State Theory, Carbene, Tunnelling
\end{abstract}

\section{Introduction}

Tunnelling in the reactivity of carbenes has been the focus of much attention in recent years, particularly in the context of tunnelling controlled reaction pathways[1-3], and due to their importance in organic synthesis[4]. The reactions of cyclocarbenes, and the effects of their 5 substituents have also been the subject of much theoretical scrutiny[5-9].

In order to study polyatomic systems, including tunnelling effects, development of efficient and reliable dynamical theories is required. One such theory is Semiclassical Transition State Theory (SCTST), as developed originally by Miller et al. [10]. This has previously been applied and tested on a variety of polyatomic reactions [11-14]. Applying the theory in reduceddimensions allows for even greater efficiency, and has been shown to be nearly as accurate as the full-dimensional treatment in many cases, despite the significant computational saving[15-19].

Email addresses: timothy.burd@chem.ox.ac.uk (Timothy A. H. Burd), xiao.shan@chem.ox.ac.uk (Xiao Shan), david.clary@chem.ox.ac.uk (David C. Clary) 


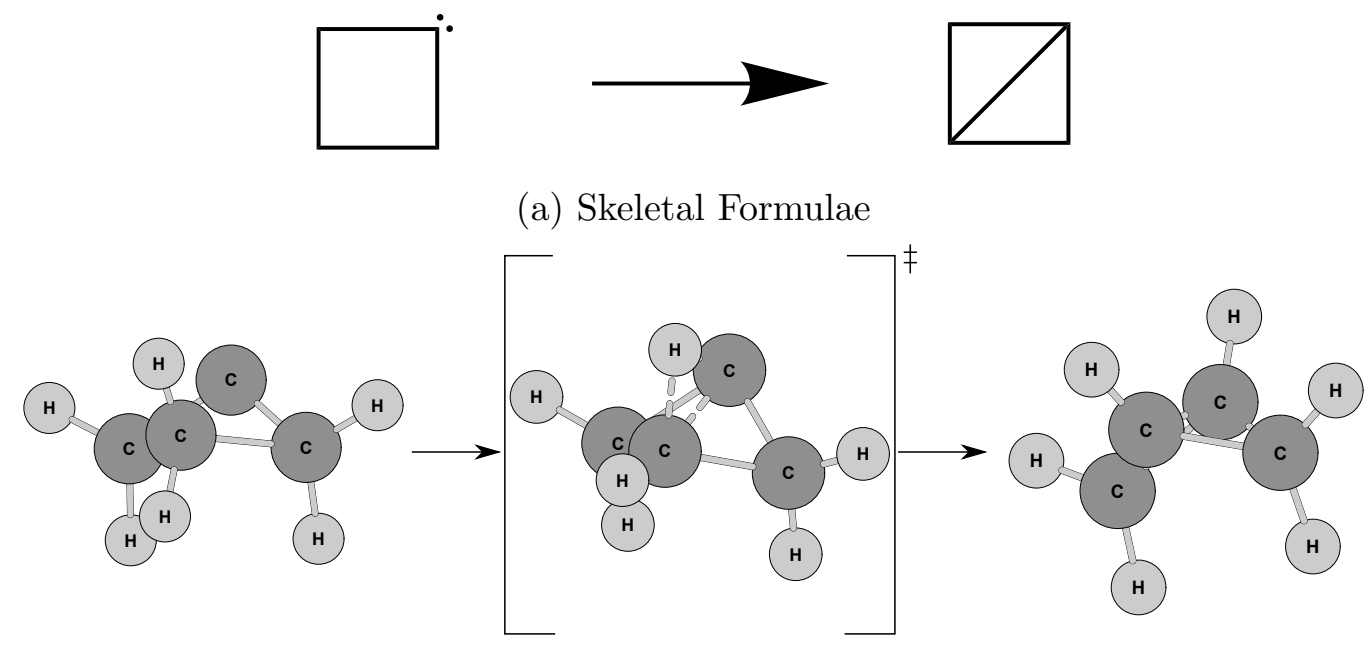

(b) 3D Structures

Figure 1: Reaction Mechanism

Here we investigate tunnelling in the unimolecular isomerisation of cyclobutylidene, shown in Fig. 1, which has previously been studied with Variational Transition State Theory (VTST) with the small curvature tunnelling (SCT) approximation[5], as well as experimentally[20]. Note that other rearrangement reactions of the reactant also occur[6]. In this work, we aim to show that SCTST gives good agreement with VTST results when using the same level of $a b$ initio theory, even at very low temperatures $(100 \mathrm{~K})$. We also determine the accuracy of a reduced-dimensional approach for this system. This provides a procedure to achieve accurate, but efficient, rate constant calculations using SCTST.

\section{Theory}

\subsection{SCTST}

A comprehensive review of the theory of SCTST in full and reduced dimensions is available in reference 15. Here we provide just a brief summary and the key theoretical results.

Within full-dimensional SCTST (FD-SCTST), the cumulative reaction probability is given by the sum over all state-dependent reaction probabilities at the energy $E_{v}$ :

$$
N\left(E_{v}\right)=\sum_{\{n\}^{\prime}} P_{\{n\}^{\prime}}\left(E_{v}\right)
$$


where $\{n\}^{\prime}$ is the set of all vibrational states of the transition state, and the state-dependent reaction probability is given by the standard WKB form:

$$
P_{\{n\}^{\prime}}\left(E_{v}\right)=\frac{1}{1+\exp \left[2 \theta_{\{n\}^{\prime}}\left(E_{v}\right)\right]}
$$

The penetration integral, $\theta$, is given analytically as a function of the frequencies $\left(\omega_{i}\right)$, anharmonic constants $\left(x_{i j}\right)$ and the vibrational quantum numbers $\left(n_{i}\right)$ of the transition state. For a system with $F$ vibrational degrees of freedom,

$$
\theta_{\{n\}^{\prime}}\left(E_{v}\right)=\pi \frac{-\Omega_{\{n\}^{\prime}}+\left[\Omega_{\{n\}^{\prime}}^{2}+4 x_{F F}\left(\Delta V_{f}+G_{0}-E_{\{n\}^{\prime}}-E_{v}\right)\right]^{1 / 2}}{2 x_{F F}} .
$$

where $\Delta V_{f}$ is the forward barrier height,

$$
\Omega_{\{n\}^{\prime}}=\frac{\hbar \omega_{F}}{i}+\sum_{i=1}^{F-1} \frac{x_{i F}}{i}\left(n_{i}+0.5\right)
$$

and

$$
E_{\{n\}^{\prime}}=\sum_{i=1}^{F-1} \hbar \omega_{i}\left(n_{i}+0.5\right)+\sum_{i \leq j}^{F-1} x_{i j}\left(n_{i}+0.5\right)\left(n_{j}+0.5\right)+G_{0} .
$$

Here $\omega_{F}$ is the imaginary reaction mode frequency, and $G_{0}$ is a barrier height correction factor, defined below. The anharmonic constants are calculated using second-order vibrational perturbation theory (VPT2) and the third and fourth order derivatives of the potential along the normal modes, $f_{i j k}$ and $f_{i i j j}$ respectively[21]:

$$
x_{i i}=\frac{\hbar^{2}}{16 \omega_{i}^{2}}\left(f_{i i i i}-\sum_{j=1}^{i} \frac{f_{i i j}^{2}}{\omega_{j}^{2}} \frac{8 \omega_{i}^{2}-3 \omega_{j}^{2}}{4 \omega_{i}^{2}-\omega_{j}^{2}}\right)
$$

$$
\begin{array}{r}
x_{i k}=\frac{\hbar^{2}}{4 \omega_{i} \omega_{k}}\left(f_{i i k k}-\sum_{j=1}^{F} \frac{f_{i i j} f_{j k k}}{\omega_{j}^{2}}\right. \\
\left.+\sum_{j=1}^{F} \frac{2 f_{i j k}^{2}\left(\omega_{i}^{2}+\omega_{k}^{2}-\omega_{j}^{2}\right)}{\left[\left(\omega_{i}+\omega_{k}\right)^{2}-\omega_{j}^{2}\right]\left[\left(\omega_{i}-\omega_{k}\right)^{2}-\omega_{j}^{2}\right]}\right), i \neq k
\end{array}
$$

and:

$$
\begin{array}{r}
G_{0}=\frac{\hbar^{2}}{64} \sum_{i=1}^{F} \frac{f_{i i i i}}{\omega_{i}^{2}}-\frac{7 \hbar^{2}}{576} \sum_{i=1}^{F} \frac{f_{i i i}}{\omega_{i}^{4}}+\frac{3 \hbar^{2}}{64} \sum_{i \neq k}^{F} \frac{f_{i i k}^{2}}{\left(4 \omega_{i}^{2}-\omega_{k}^{2}\right) \omega_{i}^{2}} \\
-\frac{\hbar^{2}}{4} \sum_{i<j<k}^{F} \frac{f_{i j k}^{2}}{\left[\left(\omega_{i}+\omega_{k}\right)^{2}-\omega_{j}^{2}\right]\left[\left(\omega_{i}-\omega_{k}\right)^{2}-\omega_{j}^{2}\right]}
\end{array}
$$


Note that special care must be taken to eliminate terms with Fermi resonances[21].

Microcanonical rate constants are then given by:

$$
k(E)=\frac{N(E)}{2 \pi \hbar \rho(E)}
$$

45

\section{2. $1 D-S C T S T$}

The limiting factor in the accuracy of many quantum calculations is the accuracy of the theory are very expensive, as they require $(2 F+1)$ Hessian calculations for each of the reactant and transition states, where $F$ is the number of vibrational degrees of freedom of the system. For systems with many atoms (such as in this work) the cost may be prohibitive at a high level of electronic structure theory.

55

In 1D-SCTST, computational expense is minimised by setting $f_{i j k}$ and $f_{i i j j}$ to zero unless $i=j=k=F$. In other words, the reaction mode is assumed to be not coupled with any other vibrational (spectator) modes in the TS, and the spectator modes are all treated harmonically. Eqs. 6 and 7 are then reduced to one single term,

$$
x_{i j}= \begin{cases}\frac{\hbar^{2}}{16 \omega_{F}^{2}}\left(f_{F F F F}-\frac{3 f_{F F F}^{2}}{5 \omega_{F}^{2}}\right) & \text { if } i=j=F \\ 0 & \text { otherwise }\end{cases}
$$

This significantly reduces the computational cost as only higher order derivatives along the reaction mode are required. These terms can be estimated numerically from inexpensive single point energy calculations, in combination with Richardson Extrapolation[23]. Here single point energies are evaluated at small displacements along the reaction coordinate either side of the transition state. Note that the FD $G_{0}$ term in Eq. 8 is also reduced to a simpler form, given by

$$
G_{0}=\frac{\hbar^{2}}{64 \omega_{F}^{2}}\left(f_{F F F F}-\frac{7 f_{F F F}}{9 \omega_{F}^{2}}\right) .
$$




\subsection{The Reduced-Dimensionality Approach}

In many systems, a FD treatment may be prohibitively expensive, whilst a one-dimensional treatment may fail to give sufficient accuracy[14]. An intermediate treatment is therefore useful. This method is often referred to as a reduced-dimensionality (RD) calculation.

For a $d$-dimensional calculation, normal modes of the transition state can be divided into $d$ active modes and $F$ - $d$ spectator modes:

$$
\left.\nu=\left\{\begin{array}{c}
\nu_{1} \\
\vdots \\
\nu_{F-d} \\
\nu_{F-d+1} \\
\vdots \\
\nu_{F}
\end{array}\right)\right\} \text { Active Modes }
$$

Spectator modes are treated harmonically, similar to a 1D-SCTST calculation, and so only contribute to the rate through their partition function. It should be noted that the 1D SCTST method discussed in the last section is, strictly speaking, also a RD approach. However, because it does not offer any possible selection of active modes, it is different from the RD approach we are about to introduce in this section.

In quantum scattering studies of bimolecular hydrogen transfer reactions, the active degrees of freedom are often chosen based on chemical intuition (for example, bond-breaking and bondforming degrees of freedom[24, 25]). For unimolecular reactions, or more complex bimolecular reactions, the choice is less clear. Here we describe a method to indicate the strongly coupled degrees of freedom, without requiring calculation of the full couplings (as needed for a FD 85 calculation). 
In our RD analyses, the non-reactive active modes are selected based on their anharmonic coupling terms to the reaction mode, $x_{i F}$, whose full expression was given in Eq. 7. From two Hessians displaced along the reaction mode, derivatives of the form $f_{i j F}$ and $f_{F F i i}$ (where $F$ corresponds to the reaction mode) can be calculated directly. These derivatives, along with the harmonic frequencies, give enough information to calculate approximate anharmonic constants of the form:

$$
x_{i F}^{\text {approx }}=\frac{\hbar^{2}}{4 \omega_{i} \omega_{F}}\left(f_{i i F F}+\sum_{j=1}^{F} \frac{2 f_{i j F}^{2}\left(\omega_{i}^{2}+\omega_{F}^{2}-\omega_{j}^{2}\right)}{\left[\left(\omega_{i}+\omega_{F}\right)^{2}-\omega_{j}^{2}\right]\left[\left(\omega_{i}-\omega_{F}\right)^{2}-\omega_{j}^{2}\right]}\right)
$$

which serves as an approximation to the full expression:

$$
x_{i F}=\frac{\hbar^{2}}{4 \omega_{i} \omega_{F}}\left(f_{i i F F}-\sum_{j=1}^{F} \frac{f_{i i j} f_{j F F}}{\omega_{j}^{2}}+\sum_{j=1}^{F} \frac{2 f_{i j F}^{2}\left(\omega_{i}^{2}+\omega_{F}^{2}-\omega_{j}^{2}\right)}{\left[\left(\omega_{i}+\omega_{F}\right)^{2}-\omega_{j}^{2}\right]\left[\left(\omega_{i}-\omega_{F}\right)^{2}-\omega_{j}^{2}\right]}\right)
$$

where the second term (containing derivatives of the form $f_{i i j}$ ) is not present in $x_{i F}^{\text {approx }}$.

From these values, 'candidate modes' can be identified as those with large couplings. By taking Hessians along the candidate modes (2 Hessians per mode), the estimates can be refined and the accurate $x_{i F}$ term can be calculated using equation 13. This method thus yields accurate values of the anharmonic constants $x_{i j}$ where $i$ and $j$ are active modes, which can then be included in the SCTST calculation. For RD calculations in which $d<<F$, the reactant state can be approximated as harmonic, as in 1D-SCTST (for reactions where $\mathrm{d}$ is required to be large, this approximation is not valid, and anharmonicity in the reactant must be accounted for).

Following the above procedure, in a case of $n$ candidate modes, a total of $2 n$ Hessian calculations are required in addition to a standard TST. A special case in the RD SCTST methods is 1D SCTST, which can have two interpretations - either as described in section 2.2 (in which all coupling to the reaction mode is neglected), or as an extension of the RD method (where coupling to other modes is partially accounted for by taking two Hessians displaced along the reaction mode). They differ just in whether $x_{F F}$ is evaluated using equation 10 or 6 . In this work (and to be consistent with previous work) we will refer to the fully-decoupled method as 1D-SCTST, and the two-hessian version as (1D+)-SCTST. This 1D+ method has been shown 
previously[14] to yield better results than the simple 1D SCTST.

\subsection{Computational Methods}

In order to compare with VTST/SCT calculations[5], we performed ab initio calculations using Gaussian 09[26] with the M06-2X DFT functional[27] and the 6-311+G(d,p) basis set[28]. SCTST calculations were performed using our in-house pySCTST code. Full details of the SCTST method can be found in reference 15. In the 1D calculations, 8 single point energies were calculated along the reaction mode, away from the TS, with a step size of $\delta Q=0.02 a_{0} \mathrm{Da}^{0.5}$ in order to determine the anharmonicity of the reaction path, calculated using Richardson extrapolation. For FD-SCTST calculations, anharmonic constants were determined using the freq=anharmonic keyword in Gaussian.

\section{Results}

\subsection{FD and $1 D$ SCTST}

We first compare FD- and 1D-SCTST to VTST/SCT calculations on cyclobutylidene[5] at the same level of quantum theory. SCTST parameters for this reaction are shown in Table 1. The level of theory used was chosen to replicate previous calculations, but the barrier heights agree within $6 \%$ of our calculations at the much higher CCSD(T) / cc-pVTZ // MP2/cc-pVTZ level of theory. The CCSD(T) barrier height was $57.8 \mathrm{~kJ} /$ mol, compared to the DFT barrier of $61.1 \mathrm{~kJ} / \mathrm{mol}$. The value of $x_{F F}$ is seen to differ greatly between its $1 \mathrm{D}$ and FD expression. Such extreme behaviour has not been seen in other systems[14, 15], and is likely to lead to a large error in the $1 \mathrm{D}$ result.

Table 1: SCTST parameters for the isomerisation of cyclobutylidene

\begin{tabular}{|cccc|}
\hline$V_{0} /(\mathrm{kJ} / \mathrm{mol})$ & $\omega_{F} / \boldsymbol{i} \mathrm{cm}^{-1}$ & $x_{F F}^{1 D} / \mathrm{cm}^{-1}$ & $x_{F F}^{F D} / \mathrm{cm}^{-1}$ \\
\hline 61.16 & 823.08 & -34.47 & -1.830 \\
\hline
\end{tabular}

The results of these rate calculations are shown in Fig. 2, which shows that whilst 1DSCTST fails to accurately capture the low-temperature behaviour of the system, FD-SCTST 

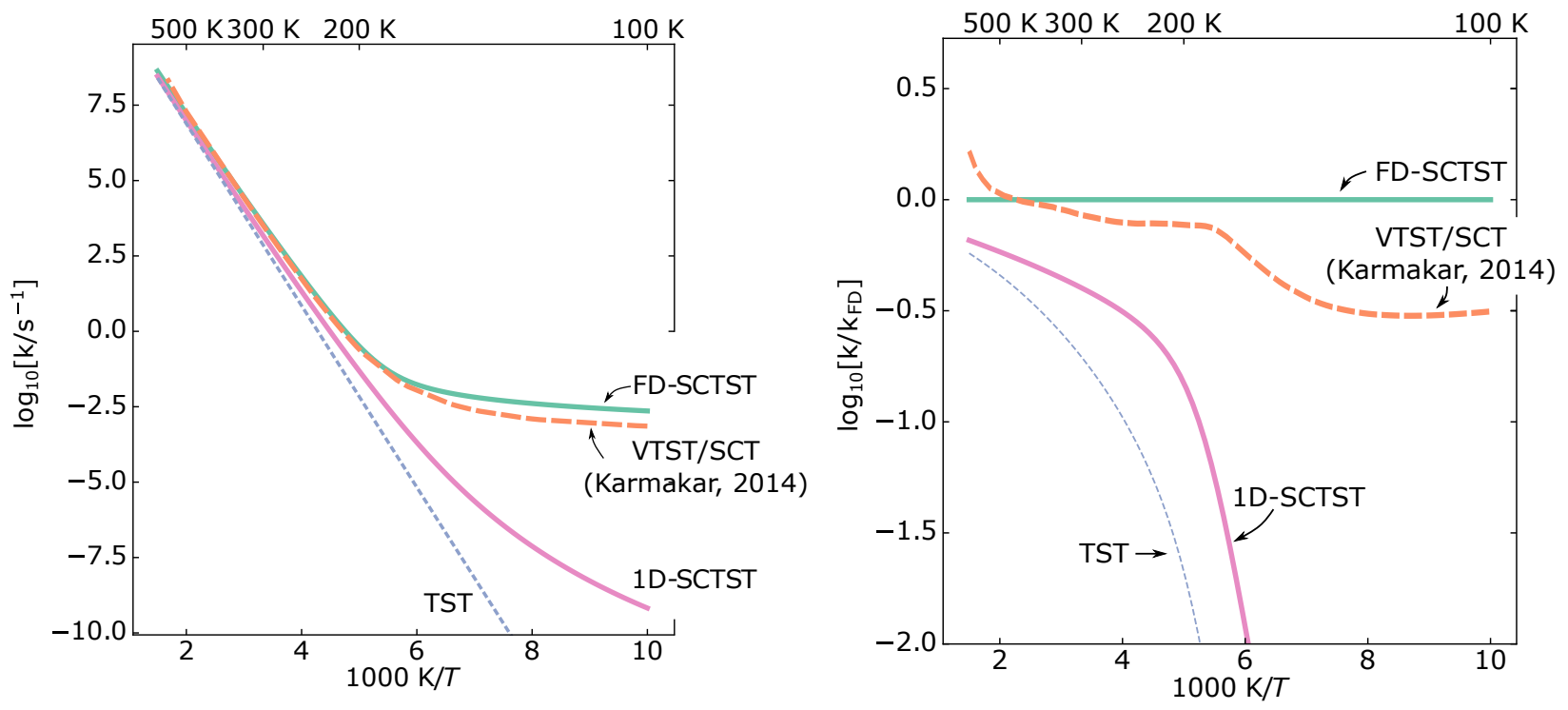

Figure 2: FD-SCTST has excellent agreement with CVT-SCT calculations by Karmakar et al. for the unimolecular isomerisation of cyclobutylidene [5]

is in good agreement with the VTST/SCT results over the whole temperature range; they differ by a factor of three at $100 \mathrm{~K}$, but less than $20 \%$ above $200 \mathrm{~K}$. 1D-SCTST underestimates the rate by several orders of magnitude at lower temperatures. The impressive agreement between VTST and SCTST further supports the conclusion that SCTST is able to pick up corner-cutting effects accurately[15], since VTST/SCT accounts for this explicitly. The near temperature-independence of the rate at low temperature is commonly observed in unimolecular tunnelling systems[29-31], but is not picked up in the 1D treatment as its very negative value of $x_{F F}$ gives a quantitatively incorrect barrier shape in the deep tunneling regime, disallowing tunnelling from the bottom of the reactant potential energy well. The FD-SCTST calculation, however, required 49 Hessian calculations at the TS.

\section{Conclusions}

\subsection{RD-SCTST}

To perform the RD calculation, the key coupled modes in the transition state must be identified. Table 2 shows how the approximate $x_{i F}$ values change as Hessians are added. This is also illustrated in Figure 3, including a direct comparison of the approximate and accurate 

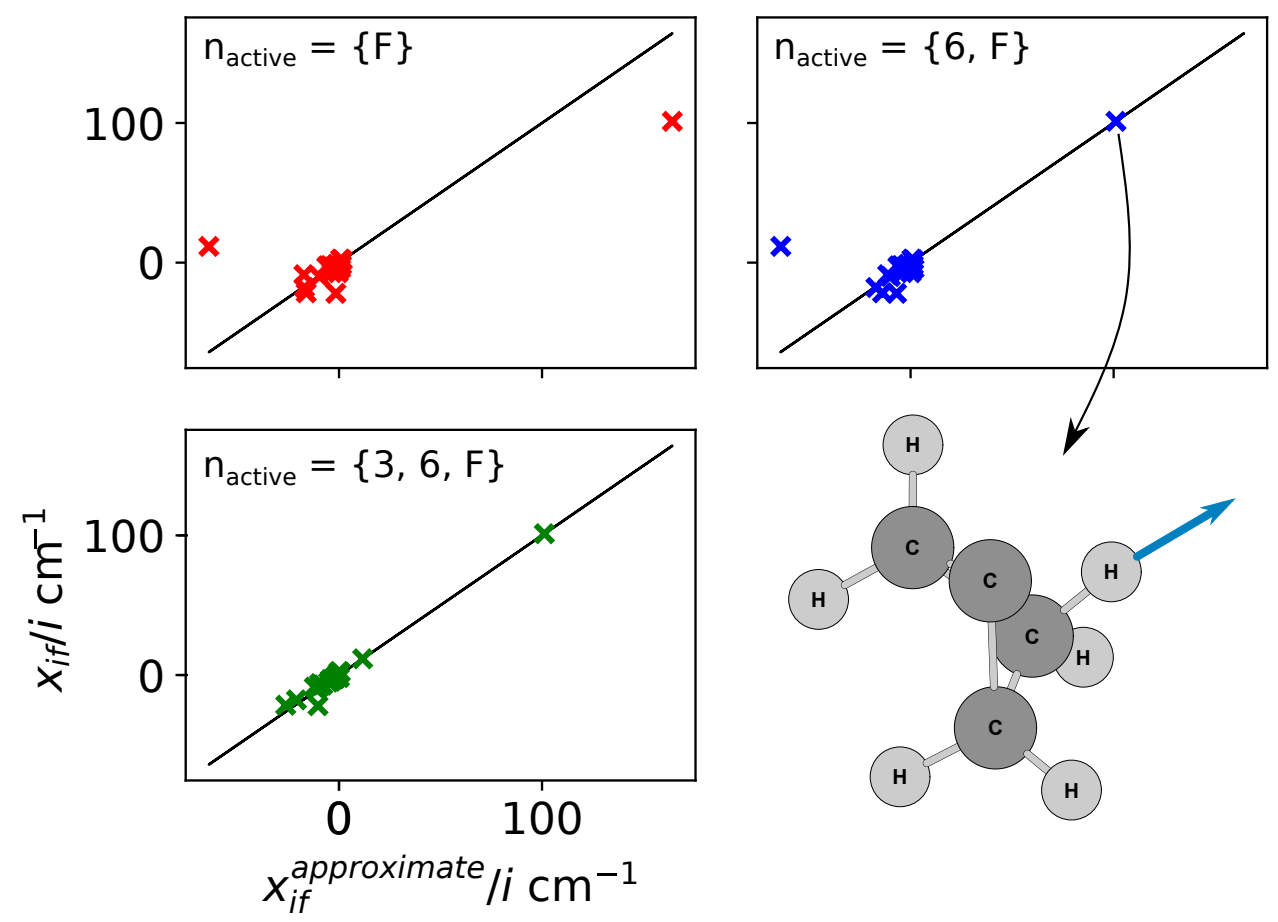

Figure 3: Determining the key modes in cyclobutylidene. Comparison of the approximate values of $x_{i F}$ with their accurate values (from a full-dimensional VPT2 calculation), as more Hessians are added. Only one bound mode is found to couple significantly to the reaction mode.. These data are tabulated in Table 2

values of $x_{i F}$ (calculated previously for the FD calculation).

In this case, the initial approximation identifies two candidate modes (modes 3 and 6), with $x_{i F}^{\text {approx }}$ values of $-64 \mathrm{i}$ and $164 \mathrm{i} \mathrm{cm}^{-1}$ respectively (shown in the top left of Fig. 3). Also, including Hessians displaced along mode 6 (top-right) then allows for accurate calculation of $x_{6 F}$ as $101 \mathrm{~cm}^{-1}$. Mode number 6 is illustrated in the bottom right of Fig. 3, and corresponds to movement of the transferring $\mathrm{H}$ atom perpendicular to the reaction path, out of the ring. Including Hessians along mode 3, however, shows a significant change in the value of $x_{3 F}$ to $+11.6 \mathrm{i} \mathrm{cm}^{-1}$. This places this mode within the grouping of other (insignificant) modes, and thus was discounted from the rest of the calculation. The possibility of large shifts between approximate and accurate $x_{i F}$ values is why the approximate $x_{i F} \mathrm{~s}$ are not, themselves, suitable for use in an SCTST calculation. Note that the accurate values of $x_{i F}$ are shown in Fig. 3 to illustrate the accuracy of the RD method, but are not required to perform the RD analysis. 
Table 2: How the approximate $x_{i F}$ s compare to the (accurate) FD value, as more Hessians are added. Colored cells show the 'active' modes in each calculation. These data are shown schematically in Fig. 3. The computational cost in a calculation is indicated as the number of Hessian matrices required.

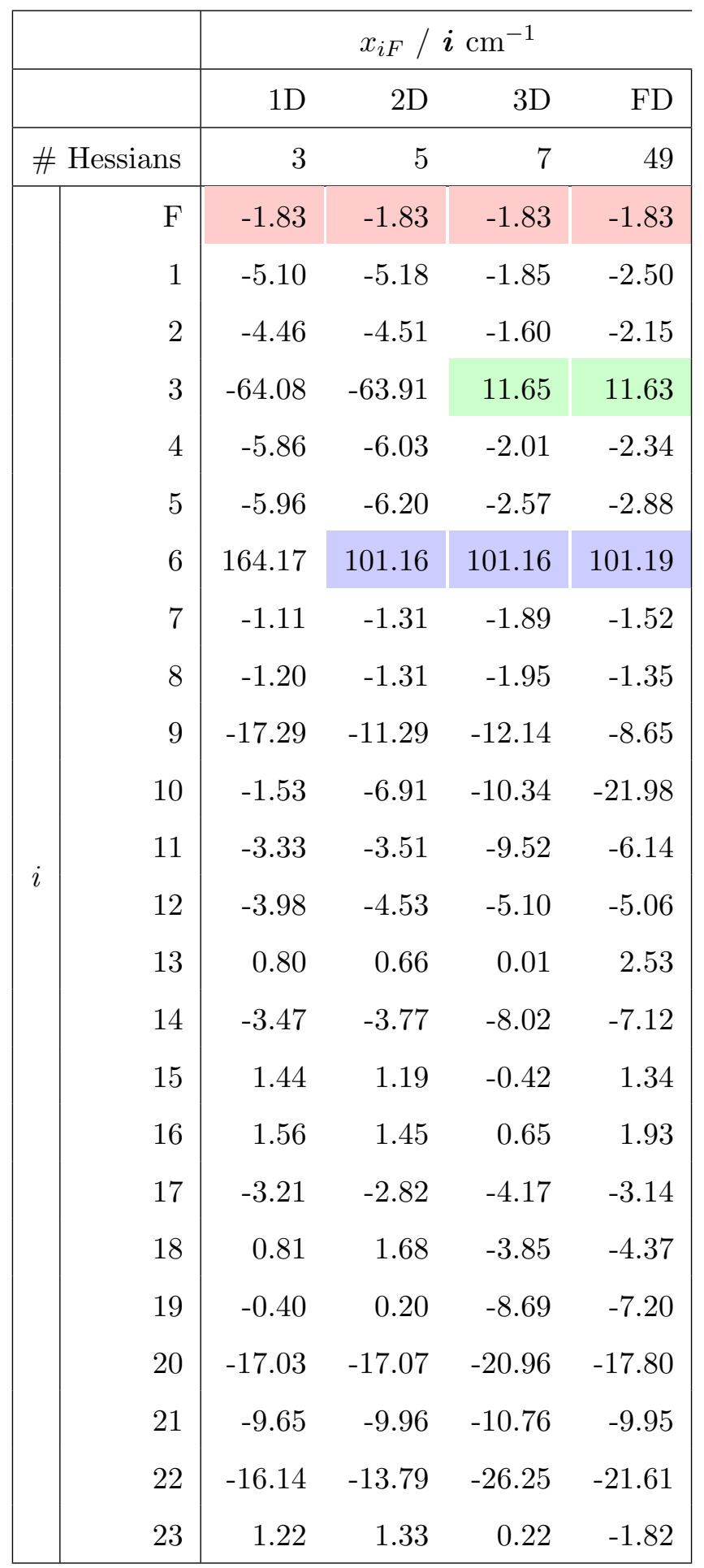



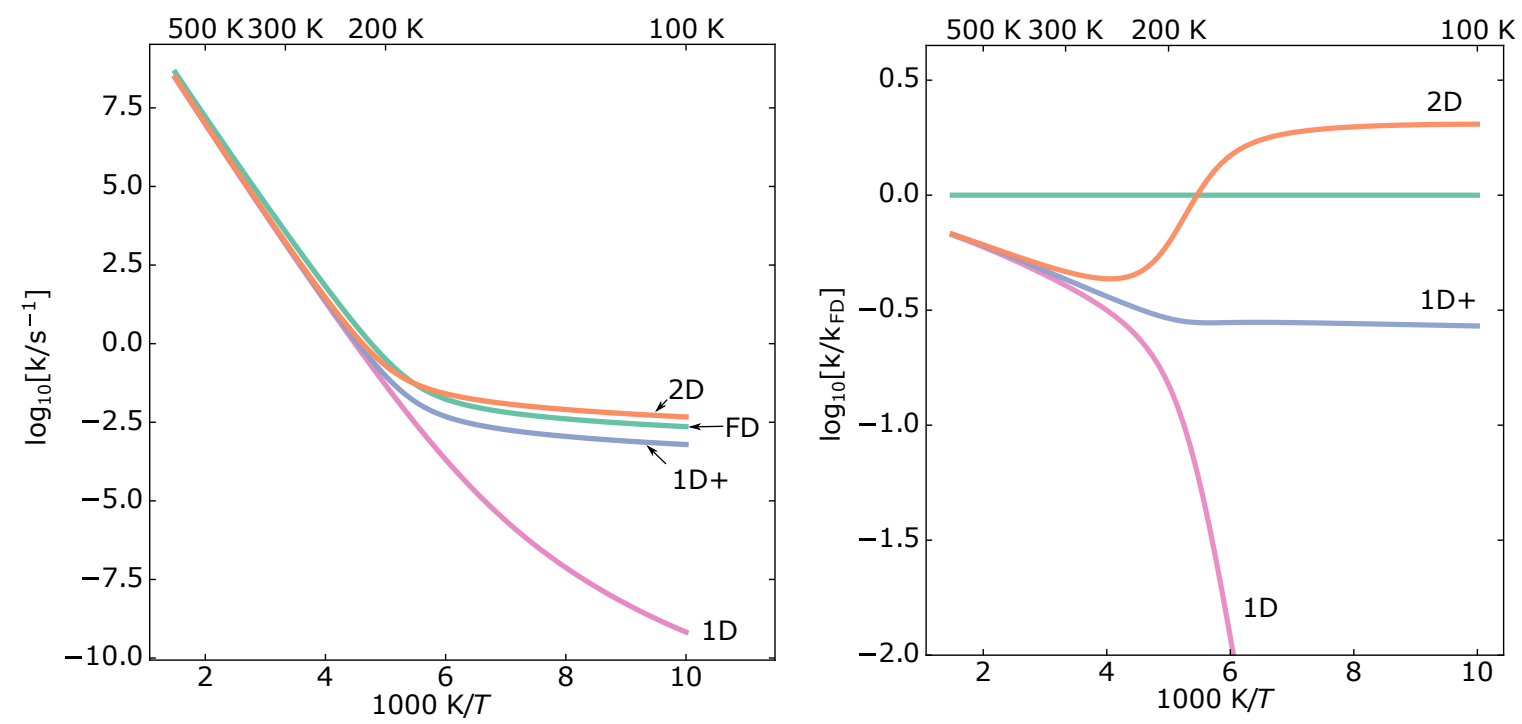

Figure 4: 2D-SCTST is an excellent approximation to the FD result in this case, whilst the 1D+ method is a significant improvement to the simple 1D-SCTST

The rate was then calculated in two-dimensions, as described previously. This calculation thus required a total of 7 Hessians.

In Fig. 4 we compare this 2D-SCTST result to the FD-SCTST calculation above, and the

\section{Conclusions}

We have described a reduced-dimensional approach to semiclassical transition state theory, and applied it to the isomerisation of cyclobutylidene. We have found that SCTST in full-dimensions gives excellent agreement with previous VTST/SCT calculations, however 1D-SCTST fails to give a good estimate of the rate at low temperatures. The reduceddimensionality scheme shows that a two-dimensional approach yields excellent agreement with 
FD-SCTST and thus describes a very efficient method to determine rate constants.

\section{Acknowledgements}

T. A. H. Burd is grateful for support from the EPSRC Centre for Doctoral Training in

Theory and Modelling in Chemical Sciences (Project Grant No. EP/L015722/1). X. Shan and D. C. Clary acknowledge financial support from the Leverhulme Trust (Project Grant No. RPG-2013-321).

\section{Supplimentary Information}

Full rate constant data are provided in the supplementary information.

\section{References}

[1] P. R. Schreiner, Tunneling Control of Chemical Reactions: The Third Reactivity Paradigm, J. Am. Chem. Soc. 139 (43) (2017) 15276-15283. doi:10.1021/jacs.7b06035.

[2] P. R. Schreiner, H. P. Reisenauer, D. Ley, D. Gerbig, C.-H. Wu, W. D. Allen, Methylhydroxycarbene: Tunneling Control of a Chemical Reaction, Science 332 (6035) (2011) 1300-1303. doi:10.1126/science.1203761.

[3] A. K. Eckhardt, D. Gerbig, P. R. Schreiner, Heavy Atom Secondary Kinetic Isotope Effect on H-Tunneling, J. Phys. Chem. A 122 (5) (2018) 1488-1495. doi:10.1021/acs.jpca.7b12118.

[4] S. R. Yetra, S. Mondal, S. Mukherjee, R. G. Gonnade, A. T. Biju, Enantioselective Synthesis of Spirocyclohexadienones by NHC-Catalyzed Formal [3+3] Annulation Reaction of Enals, Angewandte Chemie International Edition 55 (1) (2016) 268-272. doi:10.1002/anie.201507802.

[5] S. Karmakar, A. Datta, Role of Quantum Mechanical Tunneling on the $\gamma$-Effect of Silicon on Carbenes in 3-Trimethylsilylcyclobutylidene, J. Phys. Chem. B 118 (9) (2014) 25532558. doi:10.1021/jp4116029.

[6] H. M. Sulzbach, M. S. Platz, H. F. Schaefer, C. M. Hadad, Hydrogen Migration vs Carbon Migration in Dialkylcarbenes. A Study of the Preferred Product in the 
Carbene Rearrangements of Ethylmethylcarbene, Cyclobutylidene, 2-Norbornylidene, and 2-Bicyclo[2.1.1]hexylidene, J. Am. Chem. Soc. 119 (24) (1997) 5682-5689. doi:10.1021/ja970181d.

[7] G. M. Kaufman, J. A. Smith, G. G. V. Stouw, H. Shechter, Pyrolysis of Salts of p-Tosylhydrazones. Simple Methods for Preparing Diazo Compounds and Effecting Their Carbenic Decomposition, J. Am. Chem. Soc. 87 (4) (1965) 935-937. doi:10.1021/ja01082a058.

[8] L. Friedman, H. Shechter, Rearrangement and Fragmentation Reactions in Carbenoid Decomposition of Diazo Hydrocarbons, J. Am. Chem. Soc. 82 (4) (1960) 1002-1003. doi:10.1021/ja01489a063.

[9] J. P. Pezacki, D. L. Pole, J. Warkentin, T. Chen, F. Ford, J. P. Toscano, J. Fell, M. S. Platz, Laser Flash and Dual Wavelength Photolysis of 3,4-Diaza-2,2-dimethoxy1-oxa[4.5]spirooct-3-ene. Migration of Hydrogen and Carbon in Cyclobutylidene and in the Excited State of Its Precursor, J. Am. Chem. Soc. 119 (13) (1997) 3191-3192. doi:10.1021/ja961933r.

[10] W. H. Miller, Semiclassical limit of quantum mechanical transition state theory for nonseparable systems, J. Chem. Phys. 62 (5) (1975) 1899-1906. doi:10.1063/1.430676.

[11] T. L. Nguyen, J. F. Stanton, J. R. Barker, Ab Initio Reaction Rate Constants Computed Using Semiclassical Transition-State Theory: $\mathrm{HO}+\mathrm{H}_{2} \rightarrow \mathrm{H}_{2} \mathrm{O}+\mathrm{H}$ and isotopologues, J. Phys. Chem. A 115 (20) (2011) 5118-5126. doi:10.1021/jp2022743.

[12] J. R. Barker, T. L. Nguyen, J. F. Stanton, Kinetic Isotope Effects for $\mathrm{Cl}+\mathrm{CH}_{4} \rightleftharpoons \mathrm{HCl}$ $+\mathrm{CH}_{3}$ calculated using ab Initio Semiclassical Transition State Theory, J. Phys. Chem. A 116 (24) (2012) 6408-6419. doi:10.1021/jp212383u.

[13] R. E. Weston, T. L. Nguyen, J. F. Stanton, J. R. Barker, HO + CO Reaction Rates and H/D Kinetic Isotope Effects: Master Equation Models with ab Initio SCTST Rate Constants, J. Phys. Chem. A 117 (5) (2013) 821-835. doi:10.1021/jp311928w. 
[14] T. A. H. Burd, X. Shan, D. C. Clary, Catalysis and tunnelling in the unimolecular decay of criegee intermediates, Phys. Chem. Chem. Phys. 20 (39) (2018) 25224-25234. doi:10.1039/C8CP05021J.

[15] X. Shan, T. A. H. Burd, D. C. Clary, New Developments in Semiclassical Transition-State Theory, J. Phys. Chem. A 123 (22) (2019) 4639-4657. doi:10.1021/acs.jpca.9b01987.

[16] S. M. Greene, X. Shan, D. C. Clary, Reduced-Dimensionality Semiclassical Transition State Theory: Application to Hydrogen Atom Abstraction and Exchange Reactions of Hydrocarbons, J. Phys. Chem. A 119 (50) (2015) 12015-12027. doi:10.1021/acs.jpca.5b04379.

[17] X. Shan, M. R. Sambrook, D. C. Clary, Theoretical Study of Gas-Phase Unimolecular Decomposition of Simulants of the Nerve Agent VX, J. Phys. Chem. A 123 (1) (2019) 59-72. doi:10.1021/acs.jpca.8b10416.

[18] X. Shan, J. C. Vincent, S. Kirkpatrick, M. D. Walker, M. R. Sambrook, D. C. Clary, A Combined Theoretical and Experimental Study of Sarin (GB) Decomposition at High Temperatures, J. Phys. Chem. A 121 (33) (2017) 6200-6210. doi:10.1021/acs.jpca.7b04282.

[19] T. A. H. Burd, X. Shan, D. C. Clary, Tunnelling and the kinetic isotope effect in $\mathrm{CH}_{3}+$ $\mathrm{CH}_{4} \rightarrow \mathrm{CH}_{4}+\mathrm{CH}_{3}$ : An application of semiclassical transition state theory, Chem. Phys. Lett. 693 (2018) 88-94. doi:10.1016/j.cplett.2018.01.002.

[20] X. Creary, 3-Trimethylsilylcyclobutylidene. The $\gamma$-Effect of Silicon on Carbenes, J. Am. Chem. Soc. 135 (17) (2013) 6570-6578. doi:10.1021/ja400747u.

[21] V. Barone, Anharmonic vibrational properties by a fully automated second-order perturbative approach, J. Chem. Phys. 122 (1) (2004) 014108. doi:10.1063/1.1824881.

[22] D. G. Truhlar, B. C. Garrett, S. J. Klippenstein, Current Status of Transition-State Theory, J. Phys. Chem. 100 (31) (1996) 12771-12800. doi:10.1021/jp953748q.

[23] S. M. Greene, X. Shan, D. C. Clary, Rate constants of chemical reactions from semiclassical transition state theory in full and one dimension, J. Chem. Phys. 144 (24) (2016) 244116. doi:10.1063/1.4954840. 
[24] S. T. Banks, C. S. Tautermann, S. M. Remmert, D. C. Clary, An improved treatment of spectator mode vibrations in reduced dimensional quantum dynamics: Application to the hydrogen abstraction reactions $\mathrm{M}+\mathrm{CH}_{4}, \mathrm{H}+\mathrm{CH}_{4}, \mathrm{D}+\mathrm{CH}_{4}$, and $\mathrm{CH}_{3}+\mathrm{CH}_{4}$, J. Chem. Phys. 131 (4) (2009) 044111. doi:10.1063/1.3177380.

[25] S. M. Greene, X. Shan, D. C. Clary, An investigation of one- versus two-dimensional semiclassical transition state theory for $\mathrm{H}$ atom abstraction and exchange reactions, $\mathrm{J}$. Chem. Phys. 144 (8) (2016) 084113. doi:10.1063/1.4942161.

[26] M. J. Frisch, G. W. Trucks, H. B. Schlegel, G. E. Scuseria, M. A. Robb, J. R. Cheeseman, G. Scalmani, V. Barone, B. Mennucci, G. A. Petersson, H. Nakatsuji, M. Caricato, X. Li, H. P. Hratchian, A. F. Izmaylov, J. Bloino, G. Zheng, J. L. Sonnenberg, M. Hada, M. Ehara, K. Toyota, R. Fukuda, J. Hasegawa, M. Ishida, T. Nakajima, Y. Honda, O. Kitao, H. Nakai, T. Vreven, J. A. Montgomery, Jr., J. E. Peralta, F. Ogliaro, M. Bearpark, J. J. Heyd, E. Brothers, K. N. Kudin, V. N. Staroverov, R. Kobayashi, J. Normand, K. Raghavachari, A. Rendell, J. C. Burant, S. S. Iyengar, J. Tomasi, M. Cossi, N. Rega, J. M. Millam, M. Klene, J. E. Knox, J. B. Cross, V. Bakken, C. Adamo, J. Jaramillo, R. Gomperts, R. E. Stratmann, O. Yazyev, A. J. Austin, R. Cammi, C. Pomelli, J. W. Ochterski, R. L. Martin, K. Morokuma, V. G. Zakrzewski, G. A. Voth, P. Salvador, J. J. Dannenberg, S. Dapprich, A. D. Daniels, . Farkas, J. B. Foresman, J. V. Ortiz, J. Cioslowski, D. J. Fox, Gaussian09 Revision E.01, gaussian Inc. Wallingford CT 2009.

[27] Y. Zhao, D. G. Truhlar, The M06 suite of density functionals for main group thermochemistry, thermochemical kinetics, noncovalent interactions, excited states, and transition elements: Two new functionals and systematic testing of four M06-class functionals and 12 other functionals, Theor. Chem. Account. 120 (1) (2008) 215-241. doi:10.1007/s00214-0070310-X.

[28] T. Clark, J. Chandrasekhar, G. W. Spitznagel, P. V. R. Schleyer, Efficient diffuse functionaugmented basis sets for anion calculations. III. The 3-21+G basis set for first-row elements, Li-F, J. Comp. Chem. 4 (3) (1983) 294-301. doi:10.1002/jcc.540040303.

[29] H.-H. Limbach, J. Miguel Lopez, A. Kohen, Arrhenius curves of hydrogen transfers: Tunnel 
effects, isotope effects and effects of pre-equilibria, Philos. Trans. R. Soc. Lond. B Biol. Sci. 361 (1472) (2006) 1399-1415. doi:10.1098/rstb.2006.1872.

[30] J. Kästner, Path Length Determines the Tunneling Decay of Substituted Carbenes, Chem.: Eur. J. 19 (25) (2013) 8207-8212. doi:10.1002/chem.201203651.

[31] J. Kästner, Theory and simulation of atom tunneling in chemical reactions, Wiley Interdiscip. Rev. Comput. Mol. Sci. 4 (2) (2014) 158-168. doi:10.1002/wcms.1165. 